Supporting Information for

\title{
Elucidation of Adhesive Interaction between the Epoxy Molding Compound and $\mathrm{Cu}$ Lead Frames
}

Naoaki Tsurumi ${ }^{1,2}$ Yuta Tsuji, ${ }^{2}$ Noriyuki Masago, ${ }^{1}$ Kazunari Yoshizawa ${ }^{2 *}$

1. Research and Development Center, ROHM Co., Ltd., 21 Saiin Mizosaki-cho, Ukyo-ku, Kyoto 615-8585, Japan.

2. Institute for Materials Chemistry and Engineering and IRCCS, Kyushu University, 744

Motooka, Nishi-ku, Fukuoka 819-0395, Japan.

*To whom correspondence should be addressed.

E-mail: kazunari@ms.ifoc.kyushu-u.ac.jp

\section{Table of Contents}

1. Potential energy curve for the $\mathrm{ECN}-\mathrm{PN}$ fragment detaching from the $\mathrm{Cu}(111)$ surface obtained by the NEB calculation without dispersion correction

2. Global structure of the $\mathrm{ECN}-\mathrm{PN}$ fragment molecule spreading on the $\mathrm{Cu}_{2} \mathrm{O}(111)$ surface

3. Diversity of adhesive structures obtained from the quench dynamics simulations 


\section{Potential energy curve for the $\mathrm{ECN}-\mathrm{PN}$ fragment detaching from the $\mathrm{Cu}(111)$ surface obtained by the NEB calculation without dispersion correction.}

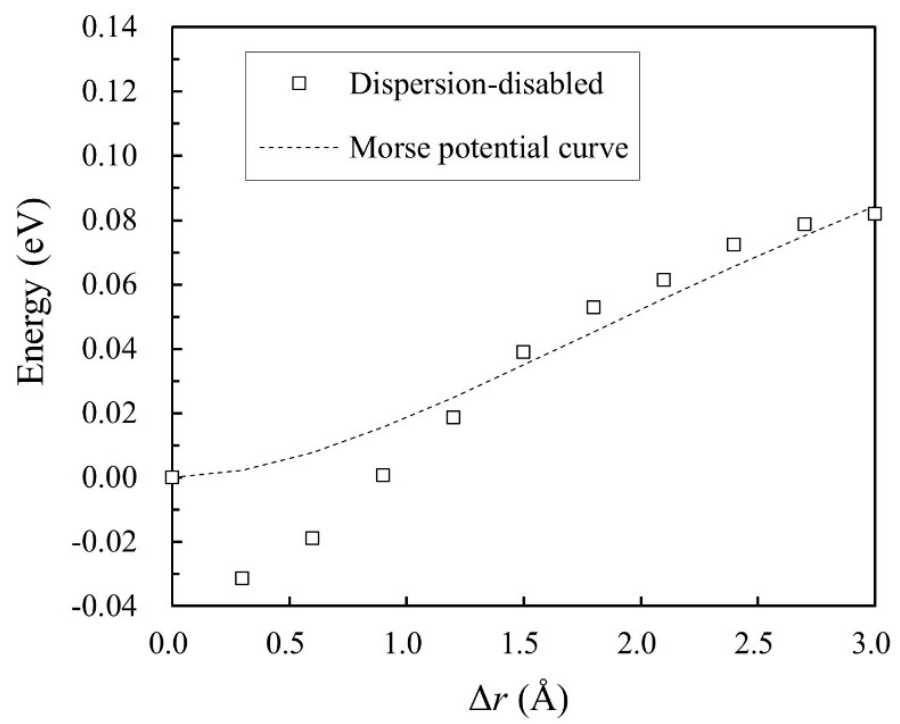

Figure S1. Enlarged view of the potential energy curve calculated without the dispersion correction for the detaching simulation of the ECN-PN fragment from the $\mathrm{Cu}(111)$ surface.

The potential energy curve obtained from the NEB calculation without dispersion force correction for the detachment of the ECN-PN fragment from the $\mathrm{Cu}(111)$ surface is enlarged in Figure S1. In the calculation of the minimum energy path by using the NEB method, it can be seen that the energy decreases when the fragment molecule is slightly pulled away from the optimized structure under the condition of without dispersion correction. This is the reason for the large error in the approximation by the least-squares method. However, in terms of the magnitude of the energy, this is not a large error. The difference between the energy of the optimized structure and that of raising the resin fragment by $0.5 \AA$ is $0.027 \mathrm{eV}$.

In addition, the structure corresponding to the point with the lowest energy in Figure S1 (second square from the left) was optimized, and the potential energy curve was calculated again using the NEB method with that structure as the initial state image. The calculation result is shown in Figure S2. The obtained energy was approximated very accurately by the Morse potential curve, and the adhesion energy was calculated to be $0.125 \mathrm{eV}$. The corresponding maximum adhesive stress is $84 \mathrm{MPa}$. Without considering the dispersion, the adhesion force to the metallic $\mathrm{Cu}$ can be concluded to be very small. 


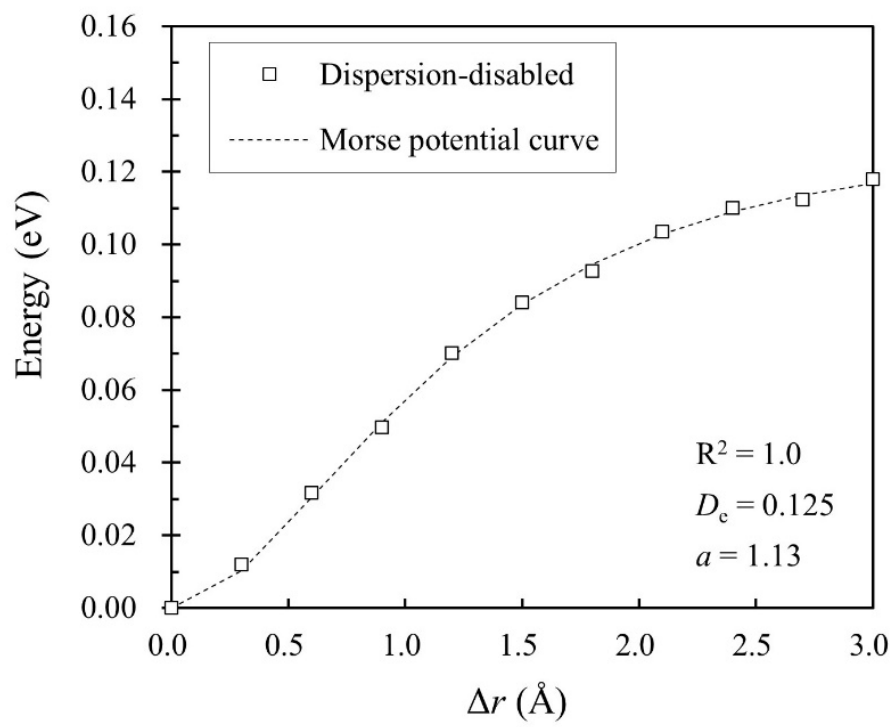

Figure S2. Recalculated potential energy curve of the ECN-PN fragment detaching from the $\mathrm{Cu}(111)$ surface without dispersion force correction. The lowest energy point in Figure S1 was reset as the optimized structure, and the potential energy was recalculated by using the NEB method. The detachment distance of the fragment molecule was set to $3 \AA$. The fitting parameters of $D_{\mathrm{e}}$ and $a$ in the Morse potential approximation are also shown, given in $\mathrm{eV}$ and $\AA^{-1}$, respectively. 


\section{Global structure of the $\mathrm{ECN}-\mathrm{PN}$ fragment molecule spreading on the $\mathrm{Cu}_{2} \mathrm{O}(111)$ surface.}

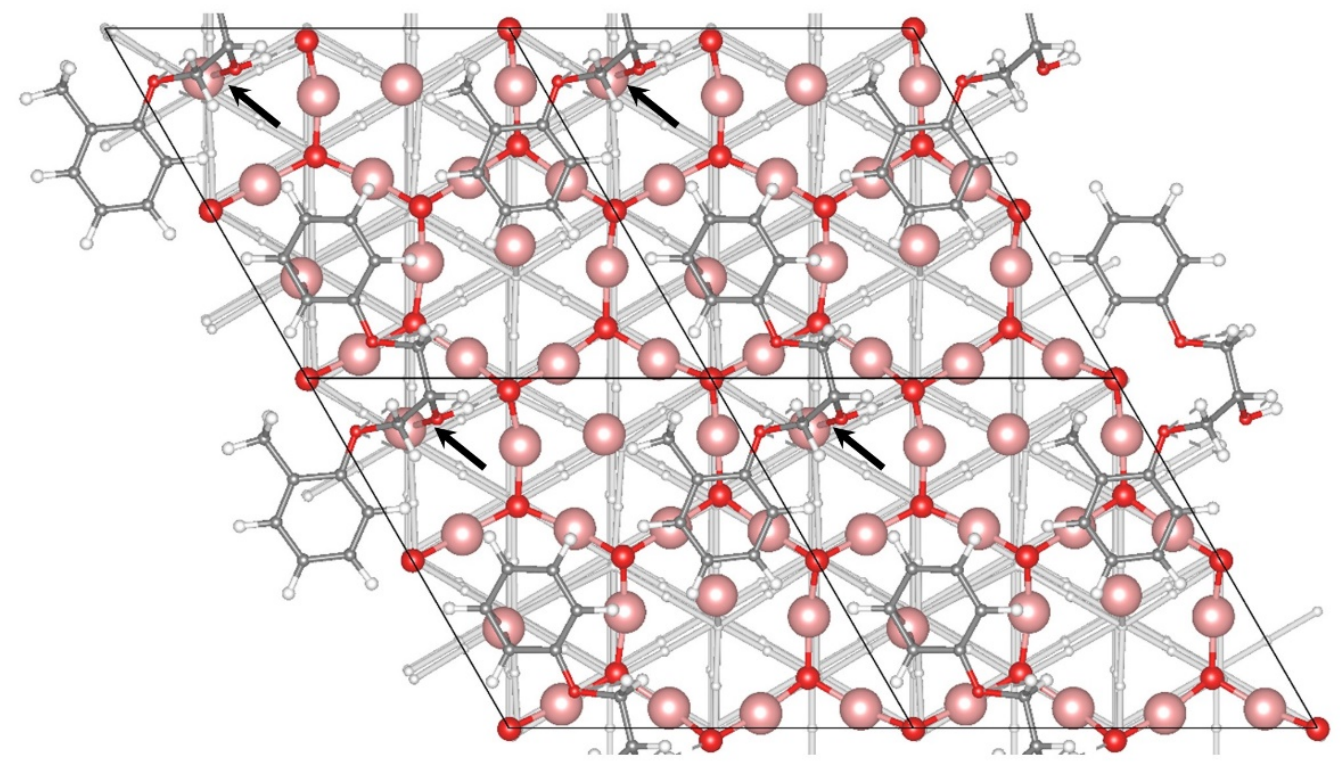

Figure S3. Global structure of the ECN-PN fragment molecule spreading on the $\mathrm{Cu}_{2} \mathrm{O}(111)$ surface is shown. The arrows correspond to the bonding sites between the $\mathrm{Cu}_{\mathrm{CUS}}$ atoms and the $\mathrm{OH}$ groups. 


\section{Diversity of adhesive structures obtained from the quench dynamics simulations.}

(a)
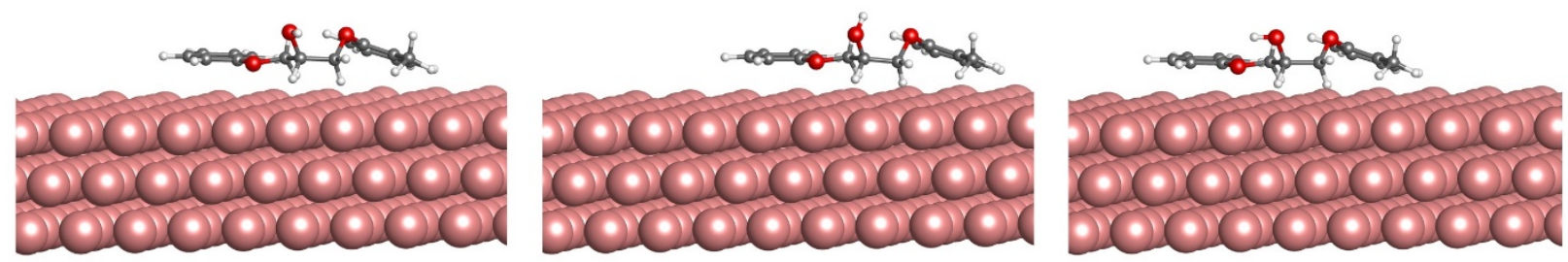

(b)
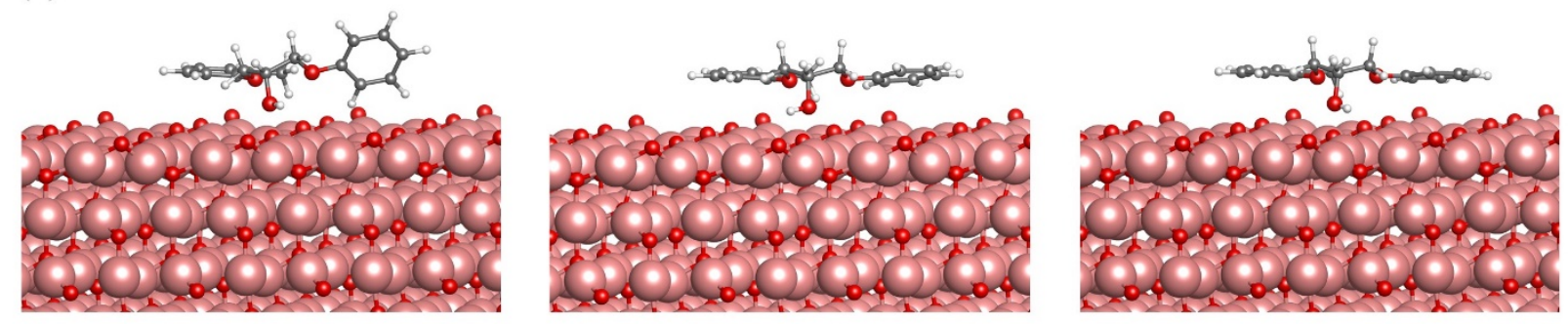

Figure S4. Various adhesive structures obtained from the quench dynamics simulation. Representative interface structures with different energies are shown from left to right. (a) The adhesion models on the $\mathrm{Cu}(111)$ surface and (b) on the $\mathrm{Cu}_{2} \mathrm{O}(111)$ surface are shown.

Figure S4 shows the fragment model structures at the adhesion interface calculated with the quench dynamics simulations. The interfacial structures on the metallic copper (Figure S4a) and on the oxidized copper (Figure S4b) with different energies are shown from left to right. As one can see, there are little change in the arrangement of the polymer fragment atoms except for the spatial positions of the functional groups. We can conclude that the essential bonding mechanism of these polymer fragments to the lead frame surface will be equivalent to the one explained in the main text based on the DFT calculations. 